\title{
Electrically-induced structure transition in nematic liquid crystal droplets with conical boundary conditions
}

\author{
V. Yu. Rudyak ${ }^{1},{ }^{*}$ M. N. Krakhalev ${ }^{2,3}$, V. S. Sutormin ${ }^{2}$, O. O. Prishchepa ${ }^{2,3}$, \\ V. Ya. Zyryanov ${ }^{2}$, J.-H. Liu ${ }^{4}$, A. V. Emelyanenko ${ }^{1}$, and A. R. Khokhlov ${ }^{1}$ \\ ${ }^{1}$ Lomonosov Moscow State University, Faculty of Physics, Moscow, 119991, Russia \\ ${ }^{2}$ Kirensky Institute of Physics, Federal Research Center - Krasnoyarsk Scientific Center, \\ Siberian Branch, Russian Academy of Sciences, Krasnoyarsk, 660036, Russia \\ ${ }^{3}$ Institute of Engineering Physics and Radio Electronics, \\ Siberian Federal University, Krasnoyarsk, 660041, Russia \\ ${ }^{4}$ Department of Chemical Engineering, National Cheng Kung University, Tainan, Taiwan
}

(Dated: August 23, 2017)

\begin{abstract}
Polymer-dispersed liquid crystal composites have been a focus of study for a long time for their unique electrooptical properties and manufacturing by "bottom-up" techniques at large scales. In this work, nematic liquid crystal oblate droplets with conical boundary conditions (CBC) under the action of electric field were studied by computer simulations and polarized optical microscopy. Droplets with CBC were shown to prefer the axial-bipolar structure, which combines a pair of boojums and circular disclination on a surface. In contrast to droplets with degenerate planar boundary conditions (PBC), hybridization of the two structure types in droplets with CBC leads to a two minima energy profile, resulting into abrupt structure transition and bistable behavior of the system. The nature of the low energy barrier in droplets with CBC makes it highly sensible to an external stimuli, such as electric or magnetic fields, temperature, light, etc. In particular, the value of the electric field of the director reorientation in the droplets with $\mathrm{CBC}$ was found to be a few times smaller than the one for droplets with PBC, and the novel droplet state remained stable after switching off the voltage.
\end{abstract}

Keywords: Nematic droplet, conical boundary conditions, electric field, director configuration, boojum, bipolar axis

Various orientation structures of the director field can be formed inside liquid crystal (LC) droplets depending on the material parameters, surface anchoring, droplet shape and size, etc. [1-7]. In turn, the optical properties of the polymer dispersed liquid crystal (PDLC) films containing such droplets are determined by orientational structure. Liquid crystal molecules are oriented by electric (or magnetic) field that results in the transformation of the orientational structure inside the droplets, and hence, in particular change of the optical properties of the whole PDLC film [8-11]. Manipulation by PDLC optical characteristics is used in displays $[12,13]$, light modulators [14], electrically-operated photonic crystals [15-17].

Peculiarities of the director reorientation within the droplets assign the response parameters (threshold voltage, switching-on and -off times, contrast ratio, etc.) of PDLC film. The target of present investigation was to find out the conditions, at which the nematic liquid crystal (NLC) droplets structure exhibits an abrupt transition at some low critical voltage applied, while below and above this critical voltage the structure remains almost unchanged.

One of the "easy" configurations, the bipolar one with two point defects-boojums, is formed at PBC (Fig. 1, a). In such droplets the bipolar axis connecting the boojums

*Electronic address: vurdizm@gmail.com is perpendicular to the short ellipsoid axis and parallel to the PDLC film plane $[9,18,19]$. When the electric field is applied along or perpendicularly to the bipolar axis, several different scenarios of the NLC droplet structure transformation can emerge. In some cases the continuous change of the orientation structure is observed with the increase of the voltage exceeding some threshold value $[9,19-21]$. In other systems the threshold process of director reorientation begins in the droplet centre and propagates gradually into the whole bulk at increasing voltage [22-24], while the location of the boojums remains invariable. In [25] the operating electric field forms initially the twist-wall coinciding with the bipolar axis and disappearing with the increasing voltage, so that the bipolar axis becomes oriented along the field. In each case, however, the NLC droplet structure exhibits a continuous transformation upon application of the electric field.

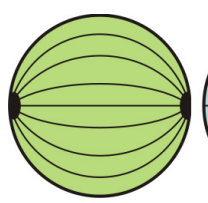

(a)

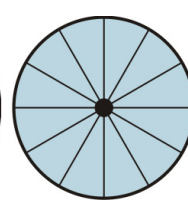

(b)

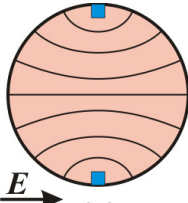

(c)

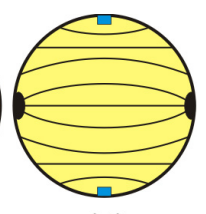

(d)
Figure 1: Director configurations in equatorial cross-section of nematic oblate droplets: (a) bipolar, (b) radial, (c) axial, and (d) axial-bipolar. 
Another "easy" configuration, the radial one (Fig. 1,b), formed at the homeotropic anchoring, has been studied in [9, 26-29]. In this configuration the NLC droplet structure does exhibit an abrupt transition, when the electric field $E$ exceeds some critical value $E_{c}$, at which the point defect is transformed into the ring one forming the axial droplet structure (Fig. 1, c) [30]. However, below this critical value, the director reorients along the field in the most part of the droplet, with simultaneous emergence of the wall perpendicular to the direction of applied voltage and crossing the point defect - hedgehog in the droplets centre. Thus, the structure does not remain unchanged below the critical value of electric field, which makes it hard to use this configuration on practice.

At the same time, the droplets with an intermediate $\mathrm{CBC}$ conditions show the most promising properties. The structures with two boojums and the ring surface defect $[7,31,32]$ or with two boojums and the point defect inside the bulk [7] are observed within such droplets. It has been shown in [33] that the bipolar axis oriented along the electric field inside the nematic droplets with two boojums and the ring defect (axial-bipolar structure, Fig. 1,d) can occur. PDLC films with conical boundary conditions at the interface have been obtained [34] recently.

In the present paper, we study the electrically-induced reorientation of the axial-bipolar structure within the oblate PDLC droplets (axes ratio $x: y: z=2: 2: 1$ ) filled with NLC. Two types of boundary conditions were examined: (i) degenerate planar anchoring as a typical experimental case, and (ii) intermediate conical anchoring, corresponding to the experimental data presented below. We use the extended Frank elastic continuum approach with Monte-Carlo annealing optimization [35] to estimate the total free energy. This approach includes the effects of the director field distortion and the formation of defects in the droplet. The interaction between the $\mathrm{LC}$ and the surface of a droplet was adopted to utilize conical surface anchoring conditions:

$$
\begin{aligned}
F= & \int_{V}\left[K_{11} / 2(\operatorname{div} \mathbf{n})^{2}+K_{22} / 2(\mathbf{n} \cdot \operatorname{rot} \mathbf{n})^{2}+\right. \\
& \left.K_{33 / 2}[\mathbf{n} \times \operatorname{rot} \mathbf{n}]^{2}+\varepsilon_{0} \Delta \varepsilon(1-(\mathbf{E} \cdot \mathbf{n}))^{2}\right] d V+ \\
& W / 2 \int_{\Sigma}\left[1-\cos ^{2}\left(\theta_{\mathbf{n k}}-\theta_{0}\right)\right] d \Sigma+F_{\text {def }},
\end{aligned}
$$

where $K_{11}, K_{22}$ and $K_{33}$ are the splay, twist and bend elasticity constants, respectively, $\mathrm{W}$ is the surface anchoring energy density, $\theta_{n k}$ is the angle between local director $\mathbf{n}$ and surface normal of the droplet $\mathbf{k}, \theta_{0}$ is the preferred angle between $\mathbf{n}$ and $\mathbf{k}$, and $F_{d e f}$ is the energy of defects calculated by the summation of the point and linear defect energies (see the details in ref. [35]). We used $\theta_{0}=90^{\circ}$ for $\mathrm{PBC}$ case and $\theta_{0}=40^{\circ}$ for CBC case, according to [34]. We produced numerical modeling of the oblate PDLC droplets filled with nematic 5CB with the ratio between long $d x=d y$ and short $d z$ eigenvectors fixed $d x / d z=2$. The ratio between elasticity constants was set to $K_{11}: K_{22}: K_{33}=1: 0.5: 1.3$ to simulate the $5 \mathrm{CB}$ nematic [36]. Linear energy density of disclination core was set to $f_{\text {line }}^{\text {core }}=10 K_{11}$ [35], and the anchoring strength $\mu=\frac{W R}{K_{11}}=10^{3}$ was used for strong anchoring. The energy-optimal structures at various values of electric field $E$ were calculated independently for two cases of surface anchoring: $\mathrm{PBC}, \theta_{0}=90^{\circ}$ (Fig. 2) and CBC, $\theta_{0}=40^{\circ}$ (Fig. 3), corresponding to the experimental data presented in this paper. Hereafter, the dimensionless notations for the electric field $e=E R\left(\varepsilon_{0} \Delta \varepsilon K_{11}^{-1}\right)^{1 / 2}$ will be used, where $R=0.5(d x d y d z)^{1 / 3}$ is the effective radius of the droplet, $\varepsilon_{0}$ is dielectric constant, and $\Delta \varepsilon$ is dielectrical anisotropy of $5 \mathrm{CB}$.

The energy-optimal structures in both cases have two symmetrical with respect to the droplet center boojums belonging to the droplet surface, while the tilt angle $\alpha$ of the bipolar axis depends on the value of electric field. In the absence of electric field the bipolar axis coincides with the long axis of ellipsoid (Fig. 2, a and Fig. 3, a). The structure remains unchanged below some critical value $e^{*}$ (Fig. 2, b and Fig. 3, b), with the director field almost undisturbed and the bipolar axis parallel to the long axis of ellipsoid (see order parameter $Q_{z z}=\left\langle\frac{1}{2}\left(3 n_{z}^{2}-1\right)\right\rangle$ and angle $\alpha$ between the bipolar axis and the long axis of ellipsoid in Fig. 2, e and Fig. 3, e). In the case of PBC $\left(\theta_{0}=90^{\circ}\right)$, there is no any disclination line, and the continuous structural transformation occurs (see vertical dash line in Fig. 2, e), at which both the director field and the bipolar axis start tilting gradually with the increasing electric field (Fig. 2, c) with maximum tilt gradient at $e^{*} \approx 5.7$ and asymptotically reaching $\alpha=\pi / 2$ at $e \rightarrow \infty$ (Fig. 2, d).

In the case of intermediate conical anchoring $\left(\theta_{0}=\right.$ $\left.40^{\circ}\right)$, the circular disclination line appears, which is nearly perpendicular to the bipolar axis at small electric field (Fig. 3, b). At $e_{c} \approx 1.75$, the discontinuous structural transformation occurs (see vertical dash line in Fig. 3, e), and both the bipolar axis and circular disclination line abruptly turn by $85^{\circ}-90^{\circ}$ (Fig. 3, c). At further increasing electric field, the bipolar axis gradually comes closer to the short axis (Fig. 3,d), asymptotically reaching $\alpha=\pi / 2$ at $e \rightarrow \infty$.

We have examined experimentally the PDLC film based on the nematic mixture LN-396 (Belarus State Technological University) and poly(isobutyl methacrylate) (PiBMA) (Sigma), assigning the CBC in this mixture with the tilt angle of director to the surface normal is $\theta=40^{\circ} \pm 4^{\circ}$ [34]. The weight ratio of $\mathrm{LN}-396$ to PiBMA was $60: 40$. The electro-optical cell was made by the TIPS method [9], at which the polymer and LC mixture was heated up to $80^{\circ} \mathrm{C}$ and then cooled down to the room temperature during one hour. The thickness of PDLC film was $25 \mu \mathrm{m}$, the LC droplets size was between 10 and $40 \mu \mathrm{m}$. The transformation of orientation 

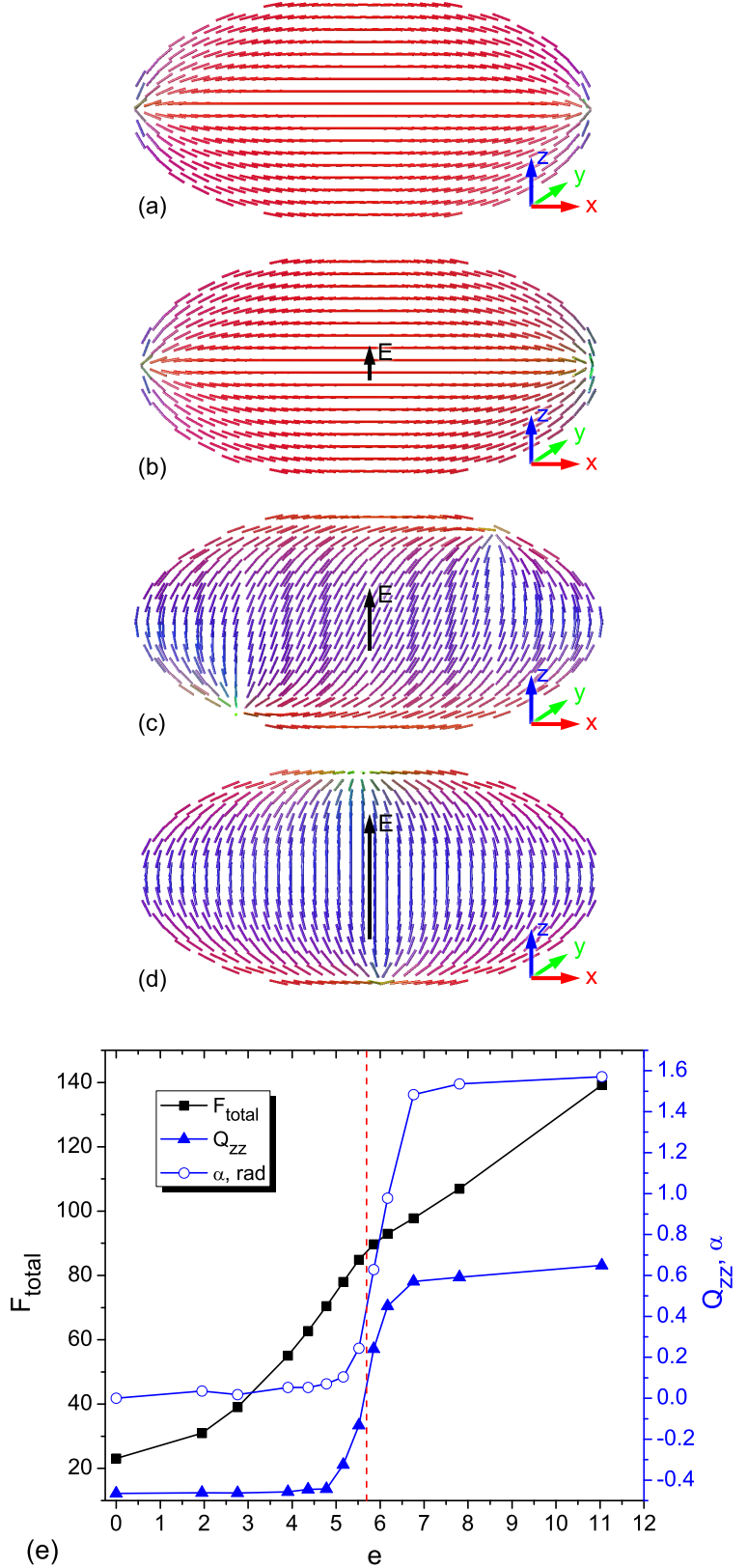

Figure 2: Droplet structures for $\theta_{0}=90^{\circ}$ at (a) $e=0$, (b) $e=3.9$, (c) $e=5.9$, and (d) $e=7.8$. Hereafter the director vectors are colored in correspondence with the direction (red along $x$-axis, green along $y$-axis, and blue along $z$-axis). (e) Dependencies of the free energy $F_{\text {total }}$, the order parameter $Q_{z z}$, and the bipolar axis tilt angle $\alpha$ on the dimensionless electric field $e$. The continuous structural transformation at $e^{*} \approx 5.7$ is marked with red dash line.

structures was studied by the polarizing optical method using the Axio Imager.A1m microscope (Carl Zeiss). Ac voltage of $1 \mathrm{kHz}$ frequency was applied to the cell under study.

The axial-bipolar structure (Fig. 4) is formed within the droplets. The bipolar axis belongs to the film plane
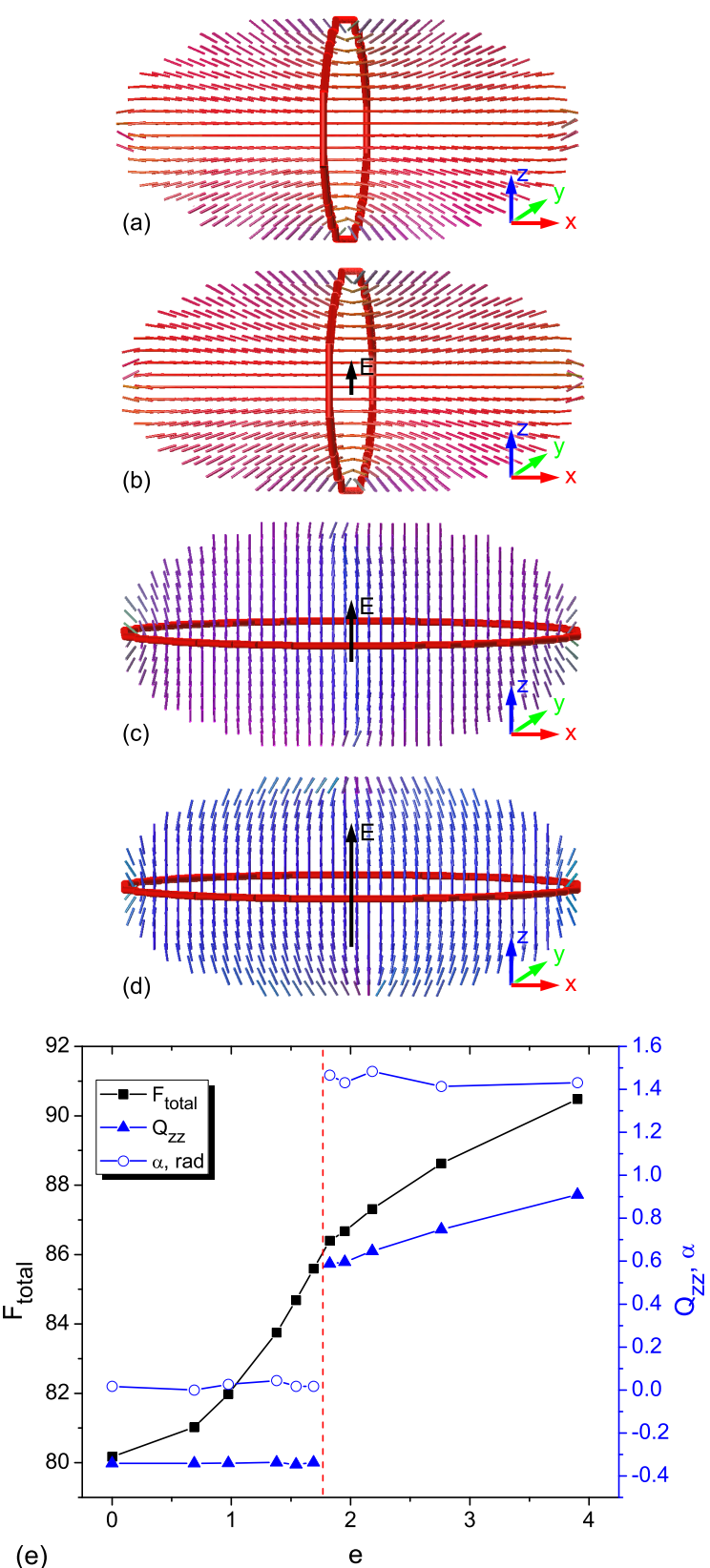

Figure 3: Droplet structures for $\theta_{0}=40^{\circ}$ at (a) $e=0$, (b) $e=$ 1.7, (c) $e=1.8$, and (d) $e=3.9$. Disclination is shown with thick red line. (e) Dependencies of the free energy $F_{\text {total }}$, the order parameter $Q_{z z}$, and the bipolar axis tilt angle $\alpha$ on the dimensionless electric field $e$. The discontinuous structural transformation at $e_{c} \approx 1.75$ is marked with red dash line.

initially (Fig. 4,a). There are no visible changes of the orientation structure at the electric field strength up to $64 \mathrm{mV} / \mu \mathrm{m}$. A slight change of the director orientation in the droplet centre starts at $72 \mathrm{mV} / \mu \mathrm{m}$ (Fig. 4,b), which is approved by deformation of the extinction bands observed in crossed polarizers. At the same time, the location of the boojums and of the circular disclination line is practically invariable. Further increase of the electric 


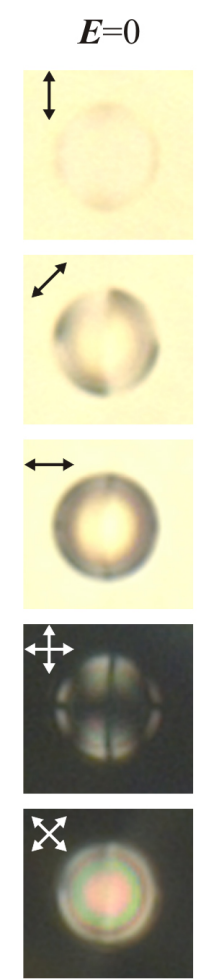

(a)
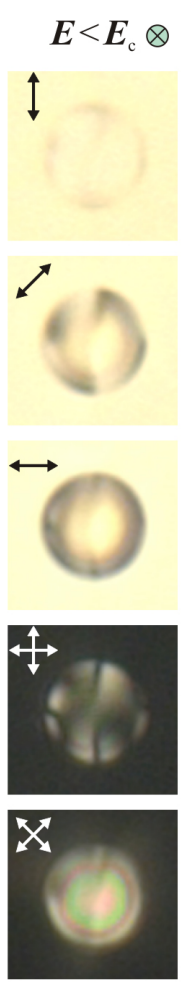

(b)
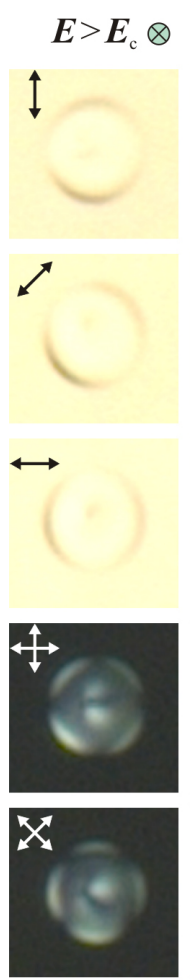

(c)
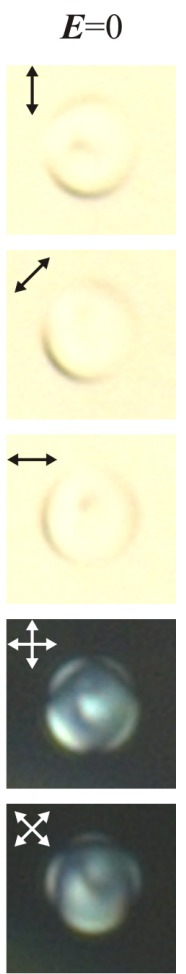

(d)

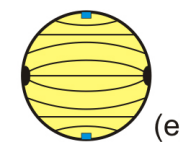

(e)

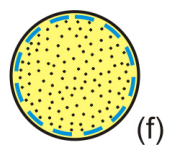

Figure 4: POM images of LC droplet: without analyser made when the polariser is perpendicular (first row), at $45^{\circ}$ angle (second row) and parallel (third row) to the bipolar axis; in the crossed polarizers, when the polariser is parallel (fourth row) and at $45^{\circ}$ angle (fifth row) to the bipolar axis. (a) Initial state $E=0$; (b) at $E=72 \mathrm{mV} / \mu \mathrm{m}$; (c) at $E=80 \mathrm{mV} / \mu \mathrm{m}$; (d) in 5 hours after switching-off. The droplet size in the film plane is $13 \mu \mathrm{m}$. The polariser directions are indicated by the double arrows. The schemes of director lines and topological defects in cross-section parallel to the film plane are presented for the voltage below (e) and above (f) the threshold value.

field up to $80 \mathrm{mV} / \mu \mathrm{m}$ results in the abrupt reorientation of the bipolar axis along the electric field, while the circular disclination line becomes oriented perpendicular to the electric field (Fig. 4,c and f). Surprisingly, the droplet structure fails to restore to the initial state, when the electric field is removed. The bipolar axis remains oriented close to the film normal and the whole structure remains the same during several hours after switching off the electric field (Fig. 4, d). In contrast to a fully reversible transition in droplets with planar anchoring, it clearly indicates the bistable behavior of the droplets with tilted anchoring.

To understand the cause of the bistability, we calculated free energy profiles of droplet with $\mathrm{CBC}$ with respect to bipolar axis tilt angle $\alpha$ (i) in absence of electric field and (ii) just above the structure transformation,

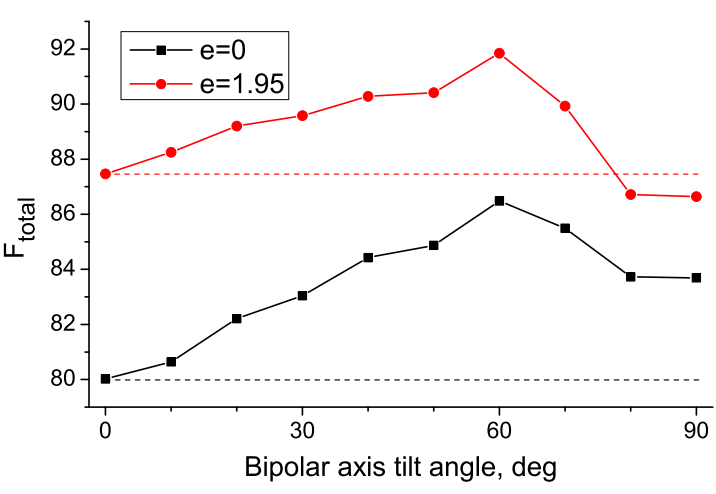

Figure 5: Dependency of the dimensionless total free energy $\left(F_{\text {total }}\right)$ of the droplet with $\theta_{0}=40^{\circ}$ on the bipolar axis tilt angle $\alpha$ at no electric field $(e=0)$ and above the structural transformation $(e=1.95)$. Dashed lines show the value of $F_{\text {total }}$ at $\alpha=0$.

$e=1.95$. For each value of $\alpha$, the position of bipolar axis was fixed, and the rest of the structure was optimized by the same Monte Carlo procedure. The results are shown in Fig. 5. In both cases, there are two minima: at $\alpha=0$ (bipolar axis is perpendicular to electric field, Fig. 3, b) and $\alpha=\pi / 2$ (bipolar axis is parallel to electric field, Fig. 3, c and d). These minima are separated by the energy barrier, which accounts for the occurrence of the discontinuous structural transformation and bistability.

To conclude, the denegerate planar and conical boundary conditions were examined for the electrically-induced structure transitions in the oblate NLC droplets, where the electric field is applied along the short principal axis of ellipsoidal droplet. To perform computer simulations, the extended Frank elastic continuum approach was expanded for the case of conical boundary conditions. In droplets with $\mathrm{PBC}$, the interplay between elastic deformations and interaction with electric field leads to the continuos director reorientation under electric field. For the dropets with CBC, the axial-bipolar structure with a pair of boojums and circular disclination line is predicted in this case, which hybridises the axial and bipolar structures of droplets with homeotropic and degenerate planar, correspondingly. In contrast to PBC droplets, in the case of $\mathrm{CBC}$ the addition of the third component, energy of disclination line, drastically changes the physics of the electrically induced structural transformation. The unique two minima potential of the bipolar axis tilt is discovered by simulations. It results into the discontinuous structural transformation with abrupt changes in structural and optical properties. The presence of two minima also explains the bistable behavior, discovered experimentally in this system. Another crucial advantage of the droplets with $\mathrm{CBC}$ is the decrease of the switchng field by miltiple times, demonstrated in both computer simulations and experiment. Finally, the studied effect of conical boundary conditions can be important in many other systems rather than just in PDLC composites. The 
high sensitivity of the droplets with $\mathrm{CBC}$ to an external stimuli (like light, temperature, shape etc) allows its use in for the perspective sensory materials of various types, including liquid solutions, isotropic-nematic solutions, and biological objects.

\section{Acknowledgments}

This study was supported by the joint project of the Russian Science Foundation (project no. 16-43-03010) and Ministry of Science and Technology of Taiwan (contract no. MOST 105-2923-E-006-007).

The simulations have been performed using the facilities of the Lomonosov Moscow State University Research Computational Center [37].
[1] T. Lopez-Leon and A. Fernandez-Nieves, "Drops and shells of liquid crystal," Colloid and Polymer Science, vol. 289, no. 4, pp. 345-359, 2011.

[2] O. O. Prishchepa, A. V. Shabanov, and V. Y. Zyryanov, "Director configurations in nematic droplets with inhomogeneous boundary conditions," Phys. Rev. E, vol. 72, p. 031712, Sep 2005.

[3] G. H. Springer and D. A. Higgins, "Toroidal droplet formation in polymer-dispersed liquid crystal films," Journal of the American Chemical Society, vol. 122, no. 28, pp. 6801-6802, 2000.

[4] E. Pairam, J. Vallamkondu, V. Koning, B. C. van Zuiden, P. W. Ellis, M. A. Bates, V. Vitelli, and A. FernandezNieves, "Stable nematic droplets with handles," Proceedings of the National Academy of Sciences, vol. 110, no. 23, pp. 9295-9300, 2013.

[5] O. Lavrentovich, "Topological defects in dispersed words and worlds around liquid crystals, or liquid crystal drops," Liquid Crystals, vol. 24, no. 1, pp. 117-126, 1998.

[6] S. A. Shvetsov, A. V. Emelyanenko, N. I. Boiko, J.H. Liu, and A. R. Khokhlov, "Communication: Orientational structure manipulation in nematic liquid crystal droplets induced by light excitation of azodendrimer dopant," J. Chem. Phys., vol. 146, pp. 211104, 2017.

[7] G.E. Volovik and O.D. Lavrentovich, "Topological dynamics of defects: boojums in nematic drops," JETP, vol. 58, pp. 1159-1166, 1983.

[8] J. W. Doane, N. A. Vaz, B. Wu, and S. Zumer, "Field controlled light scattering from nematic microdroplets," Applied Physics Letters, vol. 48, no. 4, pp. 269-271, 1986.

[9] P. Drzaic, Liquid Crystal Dispersion. Singapore: World Scientific, 1995.

[10] H.-S. Kitzerow, "Polymer-dispersed liquid crystals from the nematic curvilinear aligned phase to ferroelectric films," Liquid Crystals, vol. 16, no. 1, pp. 1-31, 1994.

[11] J. Doane, Liquid CrystalsApplications and Uses, vol. 1. World Scientific Publishing Company, 1990.

[12] J. W. Doane, A. Golemme, J. L. West, J. B. Whitehead Jr., and B.-G. Wu, "Polymer dispersed liquid crystals for display application," Molecular Crystals and Liquid Crystals Incorporating Nonlinear Optics, vol. 165, no. 1, pp. 511-532, 1988.

[13] N. H. Cho, P. Nayek, J. J. Lee, Y. J. Lim, J. H. Lee, S. H. Lee, H. S. Park, H. J. Lee, and H. S. Kim, "Highperformance, in-plane switching liquid crystal device utilizing an optically isotropic liquid crystal blend of nanostructured liquid crystal droplets in a polymer matrix,"
Materials Letters, vol. 153, pp. 136 - 139, 2015.

[14] T.-C. Hsu, C.-H. Lu, Y.-T. Huang, W.-P. Shih, and W.-S. Chen, "Concentric polymer-dispersed liquid crystal rings for light intensity modulation," Sensors and Actuators A: Physical, vol. 169, no. 2, pp. $341-346,2011$. Selected Papers from the 5th Asia-Pacific Conference on Transducers and Micro-Nano Technology.

[15] X. H. Sun and X. M. Tao, "Switchable photonic crystal for polymer dispersed liquid crystal," Optics $\&$ Laser Technology, vol. 43, no. 4, pp. $820-824,2011$.

[16] D. McPhail, M. Straub, and M. Gu, "Electrical tuning of three-dimensional photonic crystals using polymer dispersed liquid crystals," Applied Physics Letters, vol. 86, no. 5, p. 051103, 2005.

[17] P.-C. Wu, E.-R. Yeh, V. Y. Zyryanov, and W. Lee, "Spatial and electrical switching of defect modes in a photonic bandgap device with a polymer-dispersed liquid crystal defect layer," Opt. Express, vol. 22, pp. 20278-20283, Aug 2014.

[18] O. O. Prishchepa, A. V. Shabanov, V. Y. Zyryanov, A. M. Parshin, and V. G. Nazarov, "Friedericksz threshold field in bipolar nematic droplets with strong surface anchoring," JETP Letters, vol. 84, no. 11, pp. 607-612, 2007.

[19] P. S. Drzaic, "Polymer dispersed nematic liquid crystal for large area displays and light valves," Journal of Applied Physics, vol. 60, no. 6, pp. 2142-2148, 1986.

[20] M. Ambrožič, P. Formoso, A. Golemme, and S. Žumer, "Anchoring and droplet deformation in polymer dispersed liquid crystals: Nmr study in an electric field," Phys. Rev. E, vol. 56, pp. 1825-1832, 1997.

[21] A. V. Koval'chuk, M. V. Kurik, O. D. Lavrentovich, and V. V. Sergan, "The threshold for the hedgehog-ring structural transition in nematic drops in an alternating electric field," JETP, vol. 67, pp. 1065-1073, 1988.

[22] A. V. Shabanov, V. V. Presnyakov, V. Y. Zyryanov, and S. Y. Vetrov, "Characteristics of the process of reorientation of bipolar drops of a nematic with rigidly fixed poles," JETP Letters, vol. 67, no. 9, pp. 733-737, 1998.

[23] A. Shabanov, V. Presnyakov, V. Zyryanov, and S. Vetrov, "Bipolar nematic droplets with rigidly fixed poles in the electric field," Molecular Crystals and Liquid Crystals, vol. 321, no. 1, pp. 245-258, 1998.

[24] O. O. Prishchepa, A. M. Parshin, A. V. Shabanov, and V. Y. Zyryanov, "Magneto-optical study of Friedericksz threshold in polymer dispersed nematic liquid crystals," Molecular Crystals and Liquid Crystals, vol. 488, no. 1, 
pp. 309-316, 2008

[25] R. H. Reamey, W. Montoya, and A. Wong, "Videomicroscopy of NCAP films: the observation of lc droplets in real time," Proc. SPIE, vol. 1665, pp. 2-7, 1992.

[26] R. Ondris-Crawford, E. P. Boyko, B. G. Wagner, J. H. Erdmann, S. umer, and J. W. Doane, "Microscope textures of nematic droplets in polymer dispersed liquid crystals," Journal of Applied Physics, vol. 69, no. 9, pp. 6380-6386, 1991.

[27] S. Candau, P. L. Roy, and F. Debeauvais, "Magnetic field effects in nematic and cholesteric droplets suspended in a isotropic liquid," Molecular Crystals and Liquid Crystals, vol. 23, no. 3-4, pp. 283-297, 1973.

[28] V. Bodnar, O. Lavrentovich, and V. Pergamenshchik, "The threshold for the hedgehog-ring structural transiton in nematic drops in an alternating electric field," JETP, vol. 74 , pp. 60-67, 1992.

[29] S. Kralj and S. Zumer, "Freedericksz transitions in supra$\mu \mathrm{m}$ nematic droplets," Phys. Rev. A, vol. 45, pp. 24612470, 1992.

[30] P. Khayyatzadeh, F. Fu, and N. M. Abukhdeir, "Fielddriven dynamics of nematic microcapillaries," Phys. Rev E, vol. 92, p. 062509, 2015.

[31] Y.-K. Kim, S. V. Shiyanovskii, and O. D. Lavrentovich, "Morphogenesis of defects and tactoids during isotrop- icnematic phase transition in self-assembled lyotropic chromonic liquid crystals," Journal of Physics: Condensed Matter, vol. 25, no. 40, p. 404202, 2013.

[32] J. K. Gupta, J. S. Zimmerman, J. J. de Pablo, F. Caruso, and N. L. Abbott, "Characterization of adsorbateinduced ordering transitions of liquid crystals within monodisperse droplets," Langmuir, vol. 25, no. 16, pp. 9016-9024, 2009. PMID: 19719217.

[33] N. V. Madhusudana and K. R. Sumathy, "Nematic droplets with a new structure," Molecular Crystals and Liquid Crystals, vol. 92, no. 7, pp. 179-185, 1983.

[34] M. N. Krakhalev, O. O. Prishchepa, V. S. Sutormin, and V. Y. Zyryanov, "Director configurations in nematic droplets with tilted surface anchoring," Liquid Crystals, vol. 44, no. 2, pp. 355-363, 2017.

[35] V. Y. Rudyak, A. V. Emelyanenko, and V. A. Loiko, "Structure transitions in oblate nematic droplets," Phys. Rev. E, vol. 88, no. 5, p. 052501, 2013.

[36] G.-P. Chen, H. Takezoe, and A. Fukuda, "Determination of $K_{i}(\mathrm{i}=13)$ and $\mu_{j}(\mathrm{j}=26)$ in $5 \mathrm{cb}$ by observing the angular dependence of Rayleigh line spectral widths," Liquid Crystals, vol. 5, pp. 341-347, July 1989.

[37] "Supercomputing center of lomonosov moscow state university." http://hpc.msu.ru, 2017. 